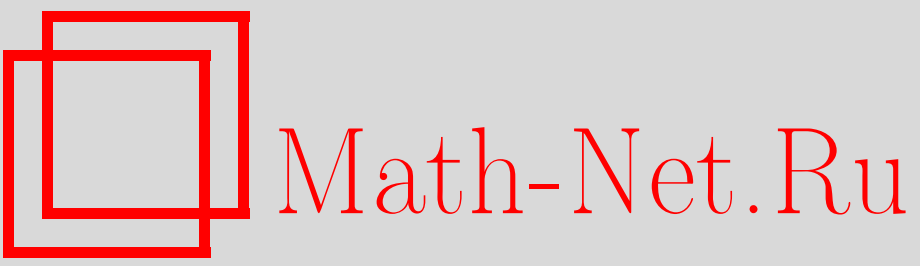

Э. А. Аринштейн, Многочастичные корреляции. Энтропия частичных распределений. Прямой вариационный метод, ТМФ, 2005, том 143, номер 1, 150-160

DOI: https://doi.org/10.4213/tmf1808

Использование Общероссийского математического портала Math-Net.Ru подразумевает, что вы прочитали и согласны с пользовательским соглашением

http://www . mathnet.ru/rus/agreement

Параметры загрузки:

IP : 54.164 .48 .24

26 апреля 2023 г., 10:06:29 
ТЕОРЕТИЧЕСКАЯ

И МАТЕМАТИЧЕСКАЯ

ФИЗИКА

Том 143, № 1

апрель, 2005

(C) 2005 r.

Э. А. Аринштейн*

\section{МНОГОЧАСТИЧНЫЕ КОРРЕЛЯЦИИ. ЭНТРОПИЯ ЧАСТИЧНЫХ РАСПРЕДЕЛЕНИЙ. ПРЯМОЙ ВАРИАЦИОННЫЙ МЕТОД}

Многочастичные корреляции классической статистической теории подчиняются вариационному принципу для функционала, являющегося на экстремали термодинамическим потенциалом. Показано, что этот функционал содержит часть, которая имеет смысл совокупности вкладов от многочастичных энтропий. Найден метод перехода от условной вариационной задачи для термодинамического потенциала к безусловной.

Ключевые слова: частичные распределения, неприводимые вклады, связные диаграммы, энтропия, прямые корреляции, полные корреляции, прямой вариационный метод.

\section{1. ВВЕДЕНИЕ}

Современная теория конденсированного состояния классических систем широко использует аппарат частичных функций распределения. Особенно это относится к теории жидкого состояния, практически полностью базируюшейся на этом методе (см. работу [1] и имеющиеся в ней ссылки).

В работе [1] было показано, что система интегральных уравнений для многочастичных плотностей (частичных функций распределения) классической статистической физики эквивалентна вариационной задаче для термодинамического потенциала как функционала от всех частичных плотностей и найден явный вид этого функционала.

Но при этом соотношение, определяюшее вспомогательный функционал (обобшенные прямые корреляции), содержало в первоначальной формулировке нефизические высшие степени макроскопического объема, которые должны быть исключены из окончательного выражения для термодинамического потенциала. Решению этой проблемы посвящена работа [2], в которой показано, что исключение подобных нефизических высших степеней объема на любой стадии преобразований может быть осушествлено переходом к разложению по связным диаграммам. Тем самым была доказана корректность найденного подхода. Но для практического решения задачи, сформулированной в работах [1], [2], необходимо решить ряд достаточно сложных проблем.

\footnotetext{
* Тюменский государственный университет, Тюмень, Россия. E-mail: earin@utmn.ru
} 
При исследовании всех приближенных уравнений теории жидкости (суперпозиционного, гиперцепного уравнений, уравнения Перкуса-Йевика) и приближений, содержащих поправки различных видов, вводимые в значительной степени произвольно, широко используются численные методы. Единственное исключение - уравнение Перкуса-Йевика для модели твердых сфер, допускаюшее аналитическое решение. Ясно, что более сложные уравнения, содержашие высшие корреляции, также необходимо исследовать не только (даже не столько) аналитически, но и численно. Однако результаты, полученные в работах [1], [2], по своему характеру являются скорее не алгоритмом расчета, а теоремой сушествования. Для того чтобы полученный вариационный принцип мог быть реализован практически, нужно иметь более детальное представление о структуре содержащихся в функционале вариационной задачи комбинаций корреляционных функций. Нужно также построить достаточно эффективный алгоритм реализации дополнительного условия, связьвающего полные и прямые корреляции.

Настояшая работа, являясь продолжением работ [1], [2], посвяшена более детальному исследованию свойств полученного разложения, и ее результаты должны привести к возможности практической реализации полученного вариационного принципа.

\section{2. ПРЕДСТАВЛЕНИЕ КОРРЕЛЯЦИОННЫХ ФУНКЦИЙ}

Для исследования совокупности частичных плотностей, корреляций и многочастичных потенциалов весьма эффективным оказывается метод производящих функционалов. Определения производящих функционалов, некоторых их свойств и операции свертки даны в работах [1], [2]; в настоящей работе используются введенные в этих работах определения и обозначения. Набор функций $x_{n}(\{n\})$ определяет функционал

$$
X(\xi)=\sum \frac{1}{n !} \int x_{n}(\{n\}) \prod_{n} \xi(i) d\{n\},
$$

функционалы $\Phi(\xi)$ и $U(\xi)=\exp \left(\int \xi(q) d q\right) \Phi(\xi)$ определены набором $n$-частичных потенциалов и потенциальных энергий в единицах $k T$, функционал $R(\xi)$ - набором $n$-частичных плотностей. Чтобы не выделять в сумме член с $n=0$, положим $\rho_{0}=1$. Кроме того, для сокрашения записи заменим $1+R(\xi)$ на $R(\xi)$. В окончательных выражениях при выполнении сверток лишняя единица исчезает.

При изучении корреляционных свойств частичных плотностей используются несколько систем корреляционных функций. Наиболее полную информацию содержат мультипликативные корреляции $M_{k}$, определяемые соотношениями

$$
\rho_{s}(\{s\})=\rho^{s} \prod\left(1+M_{1}(i)\right) \prod\left(1+M_{2}(i, k)\right) \ldots\left(1+M_{s}(\{s\})\right) .
$$

В случае газа или жидкости в отсутствии внешнего поля мультипликативная корреляция $M_{1}=0$. Функция $\ln \left(\rho_{n}(\{n\}) / \rho^{n}\right)=\sum_{k} \ln \left(1+M_{k}\right)$ является эффективной потенциальной энергией системы $n$ частиц, $\ln \left(1+M_{k}\right)$ - эффективным $k$-частичным потенциалом. Производящий функционал $\operatorname{Lr}(\xi)$ определен функциями $\ln \left(\rho_{n}(\{n\}) / \rho^{n}\right)$, функционал $\operatorname{Lm}(\xi)$ - функциями $\ln \left(1+M_{k}\right)$. Эти функционалы связаны соотношением

$$
L r(\xi)=\exp \left(\int \xi(q) d q\right) \operatorname{Lm}(\xi) .
$$


Аддитивные корреляции $g_{n}(\{n\})=\prod_{n} \rho_{1}(i) h_{n}(\{n\}) \quad\left(g_{n}\right.$ имеют размерность соответствуюшей степени плотности, $h_{n}$ безразмерны), осушествляюшие разложение $У$ рсела-Майера частичных плотностей, определяют функционал $G(\xi)$, связанный соотношением

$$
R(\xi)=\exp \left(\int \rho_{1}(q) \xi(q) d q+G\left(\rho_{1} \xi\right)\right)
$$

с функционалом $R(\xi)$. Функционал $G(\xi)$ является производящим для безразмерных аддитивных корреляций.

Можно легко установить связь между системами аддитивных и мультипликативных корреляций. По теореме о связных диаграммах имеем

$$
R(\xi)=\exp \left(\int \rho_{1}(q) \xi(q) d q+\sum_{m \geqslant 2} \frac{1}{m !} \int \prod_{m} \rho_{1}(j) \xi(j) d(j) \cdot \sum\left(\prod_{\{m\}} M_{k}\right)_{c}\right),
$$

откуда следует $h_{m}(\{m\})=\sum_{k}\left(\prod_{\{m\}} M_{k}\right)_{c}$.

Функционал $A(R)$ (или $A(\rho)$ ) вариационной задачи (термодинамический потенциал) в первоначальной формулировке имеет вид [1]

$$
\ln \frac{\rho}{z} \int \rho_{1}(q) d q+R(\xi) \otimes \Phi(\xi)-\int_{0}^{1}(R(\xi)-R(\xi \mid t)) \otimes Q(\xi ; \mu \mid t) \otimes \frac{\partial R(\mu \mid t)}{\partial t} d t
$$

где производящий функционал обобщенных прямых корреляций $Q$ определяется соотношением

$$
Q(\xi ; \eta) \otimes P(\eta ; \mu)=I(\xi \mu)-1 \equiv \exp \left(\int \xi(q) \mu(q) d q\right)-1,
$$

и

$$
P(\eta ; \mu)=R(\eta) R(\mu)-R(\eta+\mu+\eta \mu) .
$$

Вспомогательный параметр $t$ вводится таким образом, что значения $t=1$ и $t=0$ соответствуют состояниям исследуемой системы и идеального газа той же плотности. В интервале $0 \leqslant t \leqslant 1$ сохраняются все соотношения между корреляциями.

Функционалы $R(\xi)$ и $P(\xi ; \eta)$ могут быть представлены совокупностями несвязных диаграмм. Как следствие и функционал $Q(\eta ; \nu)$, и функционал $A(R)$ также представимы несвязными диаграммами. Каждая связная компонента несвязной диаграммы дает вклад, пропорциональный макроскопическому объему; несвязная диаграмма дает произведение вкладов связных компонент, что приводит к появлению высших степеней объема и необходимости проверки их сокрашения.

Переход к разложению по связным диаграммам [2] осушествляется преобразованием (для удобства у функционалов $\widetilde{P}$ и $\widetilde{Q}$ изменен знак)

$$
\begin{gathered}
R^{-1}(\eta \nu) \otimes P(\nu ; \gamma) \otimes R^{-1}(\gamma \mu)=-\exp \left(\int(\eta(q)+\mu(q)) d q\right) \widetilde{P}(\eta ; \mu), \\
\widetilde{Q}(\xi ; \eta)=-R((\xi+1) \nu) \otimes Q(\nu ; \lambda) \otimes R(\lambda(\eta+1)) .
\end{gathered}
$$


Соотношение

$$
\widetilde{Q}(\xi ; \eta) \otimes \widetilde{P}(\eta ; \mu)=I(\xi \mu)-1
$$

при этом преобразовании сохраняется, функционал вариационной задачи приобретает Вид

$$
\begin{aligned}
A(R)= & \ln \frac{\rho}{z} \int \rho_{1}(q) d q+R(\xi) \otimes \Phi(\xi)+ \\
& +\int_{0}^{1}\left(R(\xi) \otimes R^{-1}(\xi \nu \mid t) e^{-\int \nu(q) d q}-1\right) \otimes Q(\nu ; \eta \mid t) \otimes \frac{\partial L m(\eta \mid t)}{\partial t} d t .
\end{aligned}
$$

Знак тильды у модифицированных функционалов будем опускать, так как немодифицированные функционалы в дальнейшем не используются.

Модифишированный функционал $P$ по теореме о связных диаграммах приводится к виду [2]

$$
P(\xi ; \eta)=\sum_{s \geqslant 0} \frac{1}{s !} \int \frac{\prod_{s} \xi(i) \eta(i) d\{s\}}{\rho_{s}(\{s\})} d\{s\} \exp \left(\sum \frac{1}{m ! n !} S_{m, n}\right)-1
$$

где

$$
S_{m, n}=\int \sum\left(\prod M_{m^{\prime}, n^{\prime}, s^{\prime}}\right)_{c} \prod_{m} \xi(i) \prod_{n} \eta(k) d\{m+n\},
$$

причем $\left\{m^{\prime}\right\} \subseteq\{m\},\left\{m^{\prime}\right\} \neq \varnothing,\left\{n^{\prime}\right\} \subseteq\{n\},\left\{n^{\prime}\right\} \neq \varnothing,\left\{s^{\prime}\right\} \subseteq\{s\}$.

Это выражение можно преобразовать к более понятному виду, используя соотношение между связными произведениями мультипликативных корреляций и аддитивными коррелящиями. Непосредственной проверкой можно установить справедливость следующих равенств:

$$
\begin{gathered}
R_{-1}(\xi \nu) \otimes R(\nu+\mu)=\exp \left(\int\left(\xi(q)+\rho_{1}(q) \mu(q)\right) d q+\Delta_{1}\right), \\
\Delta_{1}=G\left(\xi+\rho_{1} \mu\right)-G(\xi), \\
P(\xi ; \eta)=R^{-1}(\xi \eta(1+\gamma)) \otimes_{\gamma} \exp \left(\Delta_{2}\right), \\
\Delta_{2}=\exp (G(\xi+\eta+\gamma)-G(\xi+\gamma)-G(\eta+\gamma)+G(\gamma))-1,
\end{gathered}
$$

или

$$
P(\xi ; \eta)=R^{-1}(\xi \eta)-1+P_{1}(\xi ; \eta)
$$

где

$$
P_{1}(\xi ; \eta)=R^{-1}(\xi \eta(1+\gamma)) \otimes_{\gamma}\left(\exp \left(\Delta_{2}\right)-1\right) .
$$

При этом $P_{1}(\xi ; \eta)=P_{1}(\eta ; \xi)$ и $P_{1}(\xi ; \eta)=0$ при $G=0$, следовательно, этот член мал при малости корреляций.

Исходя из того, что функционал $P(\xi ; \eta)$ содержит член $R^{-1}(\xi \eta)-1$, представим $Q(\eta ; \nu)$ в виде $Q(\eta ; \nu)=R(\eta ; \nu)-1+Q_{1}(\eta ; \nu)$. Соотношение

$$
\left(R^{-1}(\xi \eta)-1+P_{1}(\xi ; \eta)\right) \otimes\left(R(\eta ; \nu)-1+Q_{1}(\eta ; \nu)\right)=I(\xi \nu)-1
$$


для функционала $Q_{1}(\eta ; \nu)$ приобретает вид

$$
R^{-1}(\xi \eta) \otimes Q_{1}(\eta ; \nu)+P_{1}(\xi ; \mu) \otimes I(\mu \eta) \otimes Q_{1}(\eta ; \nu)=-P_{1}(\xi ; \eta) \otimes R(\eta ; \nu),
$$

где

$$
I(\mu \eta)=R(\mu ; \lambda) \otimes R^{-1}(\lambda \eta)=\exp (\lambda \eta) .
$$

Сверткой с функционалом $R$ соотношение для несимметричного функционала $R^{-1} \otimes Q_{1}$ приводится к виду

$$
Q_{1}(\eta ; \nu)+R(\eta ; \mu) \otimes P_{1}(\mu ; \xi) \otimes Q_{1}(\xi \nu)=-R(\eta ; \mu) \otimes P_{1}(\mu ; \xi) \otimes R(\xi \nu) .
$$

Эти соотношения позволяют получить очевидное представление для функционалов $R^{-1} \otimes Q_{1}$ и $Q_{1}$ в виде итерационных рядов.

Свертку некоторого произвольного функционала с произведением двух функционалов можно представить в виде

$$
A(\xi) \otimes B_{1}(\xi) B_{2}(\xi)=A\left(\xi+\xi^{\prime}\right) \otimes B_{1}\left(\xi^{\prime}\right) B_{2}(\xi)
$$

Следовательно, учитывая, что

$$
\begin{aligned}
R\left(\eta ; \mu+\mu^{\prime}\right) \otimes R^{-1}\left(\mu^{\prime} \xi(1+\gamma)\right)= & \exp \left(\int \eta(q) \xi(q)(1+\gamma(q)) d q+\int \rho_{1}(q) \eta(q) \mu(q) d q\right) \times \\
& \times \exp \left(G\left(\eta \xi(1+\gamma)+\rho_{1} \eta \mu\right)-G(\eta \xi(1+\gamma))\right),
\end{aligned}
$$

свертку $R(\eta \mu) \otimes P_{1}(\mu ; \xi)$ можно представить в виде

$$
\begin{aligned}
& \exp \left(\int \eta(q) \xi(q)(1+\gamma(q)) d q+\int \rho_{1}(q) \eta(q) \mu(q) d q+G\left(\eta \xi(1+\gamma)+\rho_{1} \eta \mu\right)-\right. \\
& -G(\eta \xi(1+\gamma))) \otimes_{\mu, \gamma} \exp (G(\xi+\mu+\gamma)-G(\xi+\gamma)-G(\mu+\gamma)+G(\gamma))= \\
& =\exp \left(\int \eta(q) \xi(q) d q+G\left(\eta \xi(1+\gamma)+\rho_{1} \eta \mu\right)-G(\eta \xi(1+\gamma))\right) \otimes_{\mu, \gamma} \\
& \otimes_{\mu, \gamma} \exp \left(G\left(\xi+\rho_{1} \eta+\xi \eta+\mu+\gamma\right)-G(\xi+\xi \eta+\gamma)-\right. \\
& \left.\quad-G\left(\rho_{1} \eta+\xi \eta+\mu+\gamma\right)+G(\xi \eta+\gamma)\right)
\end{aligned}
$$

Отсюда следует, что обобщенные прямые корреляции могут быть представлены в виде совокупности связных цепочек свернутых (переплетенных) полных корреляций и не содержат множителей вида $\rho_{n}^{-1}$, которые давали бы расходящиеся вклады при малых расстояниях между частицами. Функционал $\partial L m(\eta \mid t) / \partial t$ определяется совокупностью функций $\left(1+M_{n}(\{n\} \mid t)\right)^{-1} \partial M_{n}(\{n\} \mid t) / \partial t$, которые связывают все цепочки в единую связную диаграмму, что приводит к правильной зависимости термодинамического потенциала от объема. 


\section{3. ЭНТРОПИЯ ЧАСТИЧНЫХ РАСПРЕДЕЛЕНИЙ}

Из обшего выражения для термодинамического потенциала можно выделить член

$$
\int_{0}^{1}\left(R(\xi) \otimes R^{-1}(\xi \nu \mid t) e^{-\int \nu(q) d q}-1\right) \otimes(R(\nu \eta \mid t)-1) \otimes \frac{\partial L m(\eta \mid t)}{\partial t} d t,
$$

который соответствует члену $R(\nu \eta \mid t)-1$, выделенному из функционала $Q$.

Рассмотрим свертку $R(\xi \mid 1) \otimes R^{-1}(\xi \nu \mid t) e^{-\int \nu(q) d q} \otimes R(\nu \gamma \mid t)$. Компактноевыражение этой свертки получим, используя выражения для функционала $R$ через функционал $G$ и принимая во внимание, что экспонента от дифференциального оператора, входящего в определение свертки, является оператором сдвига. Таким образом,

$$
\begin{aligned}
R(\xi \mid 1) & \otimes R^{-1}(\xi \nu \mid t) e^{-\int \nu(q) d q} \otimes R(\nu \gamma \mid t)=e^{\int \rho_{1}(q) \xi(q) d q+G\left(\rho_{1} \xi \mid 1\right)} \otimes \\
& \otimes e^{\int\left(\xi(q)-\rho_{1}(q)\right) \gamma(q) d q+G\left(\left(\xi-\rho_{1}\right) \gamma \mid t\right)-G(\xi \gamma \mid t)}= \\
= & e^{G\left(\rho_{1}(\xi+1) \gamma \mid 1\right)} \otimes \xi e^{G(\xi \mid t)-G\left(\xi+\rho_{1} \gamma \mid t\right)} .
\end{aligned}
$$

В этом выражении отделены друг от друга корреляции, содержащие и не содержащие вспомогательный параметр $t$. Такое представление может оказаться удобным при реализации алгоритма, предусматриваюшего выполнение свертки после выполнения интегрирования по параметру.

Возможен другой порядок вычислений - проведение интегрирования по параметру $t$ после выполнения свертки. В этом случае из функционалов $G\left(\rho_{1}(\xi+1) \gamma \mid 1\right)$ и $G\left(\xi+\rho_{1} \gamma \mid t\right)$ можно выделить части, не содержащие функцию $\xi$, по которой производится свертка и, следовательно, не затрагиваемые этой сверткой. В итоге получим

$$
\begin{aligned}
R(\xi \mid 1) & \otimes R^{-1}(\xi \nu \mid t) e^{-\int \nu(q) d q} \otimes R(\nu \gamma \mid t)=e^{G\left(\rho_{1} \gamma \mid 1\right)-G\left(\rho_{1} \gamma \mid t\right)} \times \\
& \times e^{G\left(\rho_{1}(\xi+1) \gamma \mid 1\right)-G\left(\rho_{1} \gamma \mid 1\right)} \otimes_{\xi} e^{G(\xi \gamma \mid t)+G\left(\rho_{1} \gamma \mid t\right)-G\left(\xi+\rho_{1} \gamma \mid t\right)} .
\end{aligned}
$$

При этом следует иметь в виду, что при свертке полученных функционалов с функционалом $\partial \operatorname{Lm}(\gamma \mid t) / \partial t$ фактически происходит их разложение в интегростепенной ряд по функции $\gamma$.

Поэтому рассмотрим представление этого выражения, которое возникает при явном разложение по $\gamma$, что позволит выделить члены, имеющие вполне определенный физический смысл:

$$
\begin{aligned}
R(\xi \mid 1) & \otimes R^{-1}(\xi \nu \mid t) e^{-\int \nu(q) d q} \otimes R(\nu \gamma \mid t)= \\
& =R(\gamma \mid 1)+R(\xi \mid 1) \otimes R^{-1}(\xi \nu \mid t)\left(e^{-\int \nu(q) d q}-1\right) \otimes R(\nu \gamma \mid t) .
\end{aligned}
$$

Член $R(\gamma \mid 1)$, не содержащий вспомогательного параметра $t$, по которому проводится интегрирование, выделяется также при явной записи результата сверток по немым функциям $\xi$ и $\nu$ :

$$
\begin{aligned}
& \sum_{s=0}^{\infty} \sum_{n=0}^{s} \frac{(-1)^{n}}{n !(s-n) !} \int \prod_{s} \gamma(k) d(k) \rho_{s-n}(\{s-n\} \mid 1) \frac{\rho_{s}(\{s\} \mid t)}{\rho_{s-n}(\{s-n\} \mid t)}= \\
& =R(\gamma \mid 1)+\sum_{s=0}^{\infty} \sum_{n=1}^{s} \frac{(-1)^{n}}{n !(s-n) !} \int \prod_{s} \gamma(k) d(k) \rho_{s-n}(\{s-n\} \mid 1) \frac{\rho_{s}(\{s\} \mid t)}{\rho_{s-n}(\{s-n\} \mid t)}
\end{aligned}
$$


При разложении комбинаций частичных плотностей по связным произведениям коррелящий, входящих в компактные выражения, выделение члена, не содержащего вспомогательный параметр $t$, будет выглядеть следуюшим образом:

$$
\begin{aligned}
R(\xi \mid 1) & \otimes R^{-1}(\xi \nu \mid t) e^{-\int \nu(q) d q} \otimes R(\nu \gamma \mid t)= \\
& =e^{G\left(\rho_{1}(\xi+1) \gamma \mid 1\right)} \otimes_{\xi} e^{G(\xi \mid t)-G\left(\xi+\rho_{1} \gamma \mid t\right)}= \\
& =e^{-\int \rho_{1}(q) \gamma(q) d q} R(\gamma \mid 1)+e^{G\left(\rho_{1}(\xi+1) \gamma \mid 1\right)} \otimes_{\xi}\left(e^{G(\xi \mid t)-G\left(\xi+\rho_{1} \gamma \mid t\right)}-1\right) .
\end{aligned}
$$

Существенно, что выделение члена $R(\gamma \mid 1)$ в интеграле, входящем в выражение термодинамического потенциала, имеет вполне определенный физический смысл. Выражение

$$
R(\gamma \mid 1) \otimes \int_{0}^{1} \frac{\partial \operatorname{Lm}(\gamma \mid t)}{\partial t} d t=R(\gamma \mid 1) \otimes \operatorname{Lm}(\gamma \mid 1)
$$

является, очевидно, энтропией системы, определяемой частичными плотностями и мультипликативными корреляциями. Выражение

$$
\int_{0}^{1} \sum_{s=0}^{\infty} \sum_{n=1}^{s} \frac{(-1)^{n}}{n !(s-n) !} \int \prod_{s} \gamma(k) d(k) \rho_{s-n}(\cdot \mid 1) \frac{\rho_{s}(\cdot \mid t)}{\rho_{s-n}(\cdot \mid t)} \otimes \frac{\partial L m(\gamma \mid t)}{\partial t} d t
$$

определяет простейшие корреляционные поправки для термодинамического потенциала, содержашие только полные корреляционные функции. Оставшаяся часть термодинамического потенциала является функционалом не только от полных, но и от обобщенных прямых корреляций. Итерационное представление функционала $Q_{1}$ дает первый вклад

$$
-\int_{0}^{1} R(\xi \mid 1) \otimes R^{-1}(\xi \nu \mid t) e^{-\int \nu(q) d q} \otimes R(\nu \gamma \mid t) \otimes P_{1}(\mu ; \eta \mid t) \otimes R(\eta \nu \mid t) \otimes \frac{\partial L m(\nu \mid t)}{\partial t} d t
$$

и аналогичные вклады, содержашие цепочки сверток $P_{1} \otimes R$. Все компоненты этих сверток выражаются через функционалы $G$.

\section{4. ПРЯМОЙ ВАРИАЦИОННЫЙ МЕТОД}

Независимыми аргументами вариационной задачи для термодинамического потенциала являются полные корреляции, мультипликативные или аддитивные. Можно, разумеется, в качестве независимых аргументов выбрать частичные плотности, но при этом возникнет проблема учета асимптотических условий: при увеличении расстояния между двумя группами частиц обшая частичная плотность стремится к произведению плотностей этих групп, тогда как коррелящии стремятся к нулю при удалении на бесконечность хотя бы одной частицы из группы.

Однако функционал вариационной задачи содержит не только частичные плотности или полные корреляции, но также прямые корреляции, определяемые соотношениями Орнштейна-Цернике между обобщенными прямыми и полными корреляциями, т.е. дополнительными условиями. При формулировке большинства приближенных уравнений 
теории жидкости (гиперцепного, Перкуса-Йевика) и обобщений этих уравнений путем введения различных поправок также возникает проблема, связанная с существованием функций полной и прямой корреляций, которая решается путем учета не только соотношения Орнштейна-Цернике, получаюшего при этом статус исходного, но и наложения дополнительных связей, по существу произвольных, но полностью определяюших структуру приближения.

В предложенном в работах [1], [2] подходе полные корреляции определяются из вариационной задачи, соотношение Орнштейна-Цернике является дополнительным условием, определяющим прямые корреляции. Отсюда следует очевидная последовательность действий: выразить с той или иной степенью точности прямые корреляции через полные и только затем решать вариационную задачу. Однако возможен другой, технически гораздо более эффективный подход, найденный при анализе вариационной задачи для парной коррелящии [3]: рассматривать и полные, и прямые корреляции как независимые аргументы вариационной задачи, связанные дополнительным условием - обобшенным соотношением Орнштейна-Цернике (10). Свернем это соотношение с неопределенным множителем (симметричным функционалом $\Lambda(\xi ; \eta)=\Lambda(\eta ; \xi))$, проинтегрируем по параметру $t$ и вычтем полученное выражение

$$
\int_{0}^{1}\left(Q(\xi ; \nu) \otimes P(\nu ; \eta) \otimes \Lambda(\eta ; \xi) \otimes_{\xi}-\Lambda(\xi ; \xi) \otimes_{\xi}\right) d t
$$

из выражения для термодинамического потенциала (функционала $A(R)$ ). При этом очевидно, что функционал $\Lambda(\xi ; \eta)$ не содержит членов нулевого порядка: $\Lambda(\xi ; 0)=0$. Так как на экстремали дополнительное слагаемое равно нулю, то сохраним для полученного выражения прежнее обозначение, тем более, что сохраняется и его физической смысл. Но теперь полные и прямые корреляции и функционал $\Lambda$ можно рассматривать как независимые аргументы при решении вариационной задачи для функционала $A(R, Q, \Lambda)$.

Уравнение $\delta A(R, Q, \Lambda) / \delta \Lambda(\xi ; \eta)=0$ приводит, очевидно, к функциональному соотношению Орнштейна-Цернике.

Введем для краткости обозначение

$$
F(\nu ; \eta)=\left(R(\xi \mid 1) \otimes R^{-1}(\xi \nu \mid t) e^{-\int \nu(q) d q}-1\right) \frac{\partial L m(\eta \mid t)}{\partial t} .
$$

Уравнение $\delta A(R, Q, \Lambda) / \delta Q(\xi ; \eta)=0$ при использовании этого обозначения примет вид

$$
F(\xi ; \eta)-\Lambda(\xi ; \nu) \otimes P(\nu ; \eta)=0
$$

Сворачивая это уравнение с функционалом $Q(\eta ; \mu)$ и принимая во внимание функциональное соотношение Орнштейна-Цернике, получим значение неопределенного множителя $\Lambda$ :

$$
\Lambda(\xi ; \mu)=F(\xi ; \eta) \otimes Q(\eta ; \mu)
$$


что позволяет исключить его из функционала вариационной задачи. В результате этой операции получим термодинамический потенциал как функционал вариационной задачи, содержащий в качестве независимых аргументов и полные, и прямые корреляции:

$$
\begin{aligned}
A(R ; Q)= & \ln \frac{\rho}{z} \int \rho_{1}(q) d q+R(\xi) \otimes \Phi(\xi)+ \\
& +e^{G\left(\rho_{1}(\xi+1) \mu \mid 1\right)} \otimes \xi \int_{0}^{1} e^{G(\xi \mid t)-G\left(\xi+\rho_{1} \mu \mid t\right)} \otimes R^{-1}(\mu \nu) \otimes \\
& \otimes(2 Q(\nu ; \eta \mid t)-Q(\nu ; \lambda \mid t) \otimes P(\lambda ; \gamma \mid t) \otimes Q(\gamma ; \eta \mid t)) \otimes \frac{\partial L m(\eta \mid t)}{\partial t} d t .
\end{aligned}
$$

Уравнение $\delta A(R, Q) / \delta R=0$ определяет все полные корреляции, функциональное соотношение Орнштейна-Цернике появляется как уравнение $\delta A(R, Q) / \delta Q(\xi ; \eta)=0$. (Более строго, это соотношение выполняется на множестве функций, на которых функционал $F(\xi ; \eta) \neq 0$.) На множестве решений уравнения $\delta A(R, Q) / \delta Q(\xi ; \eta)=0$ выражение для термодинамического потенциала принимает прежний вид.

Учитывая структуру функционала $P$, выделим из него член $R^{-1}-1$; из функционала $Q$ выделим, соответственно, член $R-1$. В итоге получим выражение для термодинамического потенциала

$$
\begin{aligned}
A(R ; Q) & =\ln \frac{\rho}{z} \int \rho_{1}(q) d q+R(\xi) \otimes \Phi(\xi)+e^{-\int \xi(q) d q} R(\xi) \otimes L m(\xi)+ \\
& +e^{G\left(\rho_{1}(\xi+1) \mu \mid 1\right)} \otimes \xi \int_{0}^{1}\left(e^{G(\xi \mid t)-G\left(\xi+\rho_{1} \mu \mid t\right)}-1\right) \otimes \frac{\partial L m(\mu \mid t)}{\partial t} d t- \\
& -e^{G\left(\rho_{1}(\xi+1) \mu \mid 1\right)} \otimes_{\xi} \int_{0}^{1} e^{G(\xi \mid t)-G\left(\xi+\rho_{1} \mu \mid t\right)} \otimes P_{1}(\mu ; \nu) \otimes R(\nu \eta) \otimes \frac{\partial L m(\eta \mid t)}{\partial t} d t- \\
& -e^{G\left(\rho_{1}(\xi+1) \mu \mid 1\right)} \otimes \xi \int_{0}^{1} e^{G(\xi \mid t)-G\left(\xi+\rho_{1} \mu \mid t\right)} \otimes\left(P_{1}(\mu ; \nu) \otimes Q_{1}(\nu ; \gamma)+\right. \\
& +R^{-1}(\mu \nu) \otimes Q_{1}(\nu ; \lambda) \otimes P_{1}(\lambda ; \eta) \otimes R(\eta \gamma)+ \\
& +R^{-1}(\mu \nu) \otimes Q_{1}(\nu ; \lambda) \otimes R^{-1}(\lambda \eta) \otimes Q_{1}(\eta ; \gamma)+ \\
& \left.+R^{-1}(\mu \nu) \otimes Q_{1}(\nu ; \lambda) \otimes P_{1} x(\lambda \eta) \otimes Q_{1}(\eta ; \gamma)\right) \otimes \frac{\partial L m(\gamma \mid t)}{\partial t} d t .
\end{aligned}
$$

В этом выражении явно выделены члены, имеющие смысл внутренней энергии и энтропии, корреляционные поправки, не содержащие прямых корреляций, и поправки, содержашие как полные, так и прямые корреляции. И прямые, и полные корреляции являются независимыми аргументами вариационной задачи. Тот факт, что термодинамический потенциал является квадратичным функционалом от прямых корреляций, может быть эффективно использован при реализации прямых вариационных методов. При этом можно аппроксимировать либо симметричньй функционал $Q$, либо несимметричный функционал $Q \otimes R^{-1}$. Компактное выражение для функционала $P_{1} \otimes R$ было приведено выше.

Ограничение порядка учитываемых корреляций или порядка корреляционных поправок позволяет строить на основе полученного выражения для термодинамического потенциала конкретные варианты приближений различной точности. 
Разложение Урсела-Майера. Приведем разложение Урсела-Майера для парной, тройной и четверной частичных плотностей:

$$
\begin{aligned}
\rho_{2}(1,2) & =\rho_{1}(1) \rho_{1}(2)+g_{2}(1,2), \\
\rho_{3}(1,2,3) & =\rho_{1}(1) \rho_{1}(2) \rho_{1}(3)+\sum \rho_{1}(i) g_{2}(j, k)+g_{3}(1,2,3), \\
\rho_{4} & =\prod \rho_{1}+\sum \rho_{1}(i) g_{3}(j, k, l)+\sum g_{2}(i, j) g_{2}(k, l)+g_{4} .
\end{aligned}
$$

Связь аддитивных и мультипликативных корреляций. Приведем разложение трех младших аддитивных корреляций через мультипликативные:

$$
\begin{aligned}
h_{2}(1,2)= & M_{2}(1,2), \\
h_{3}(1,2,3)= & M_{2}(1,2) M_{2}(1,3)+M_{2}(1,2) M_{2}(2,3)+M_{2}(1,3) M_{2}(2,3)+ \\
& +M_{2}(1,2) M_{2}(1,3) M_{2}(2,3)+M_{3}(1,2,3) \prod\left(1+M_{2}(i, k)\right), \\
h_{4}(\{4\})= & h_{4}^{(2)}+\prod\left(1+M_{2}(i, k)\right)\left(h_{4}^{(3)}+h_{4}^{(4)}\right),
\end{aligned}
$$

где

$$
\begin{aligned}
h_{4}^{(2)}(\{4\})= & \sum M_{2}(i, j) M_{2}(i, k) M_{2}(i, l)+\sum M_{2}(i, j) M_{2}(j, k) M_{2}(k, l)+ \\
& +\prod M_{2}(i, k)\left(\sum\left(M_{2}(j, l) M_{2}(m, n)\right)^{-1}+\sum\left(M_{2}(j, l)\right)^{-1}+1\right), \\
h_{4}^{(3)}= & \sum M_{3}(i, j, k) \frac{M_{2}(i, l)}{1+M_{2}(i, l)}+\prod\left(1+M_{3}(i, j, k)\right)-\left(1+\sum M_{3}(i, j, k)\right), \\
h_{4}^{(4)}= & M_{4}(\{4\}) \prod\left(1+M_{3}(i, j, k)\right) .
\end{aligned}
$$

Суммирование распространяется на все перестановки координат, входяших в функции $h_{3}$ и $h_{4}$. Значения различных индексов в приведенных выражениях не совпадают.

Отметим, что члены в $h_{3}$, содержашие $M_{3}$, и члены в $h_{4}$, содержащие $h_{4}^{(3)}$ и $h_{4}^{(4)}$, содержат также произведение $\prod\left(1+M_{2}(i, k)\right)$, малое при $r(i, k)<r_{0}$, где $r_{0}$ - радиус “твердой” (или "почти твердой") сердцевины. Это приводит к тому, что данные члены имеют сушественное значение только на расстояниях, порядка или больших $r_{0}$.

Простейшие корреляционные поправки. Приведем простейшие корреляционные поправки низших порядков к выражению для энтропии.

Поправка второго порядка (содержашая корреляции не старше второй) имеет вид

$$
-\frac{1}{2 !} \int \rho_{1}(1) \rho_{1}(2) M_{2}(1,2) d\{2\}
$$

вне зависимости от способа введения вспомогательного параметра $t$. 
Конкретный вид старших поправок зависит от способа введения этого параметра. Очевидно, что возникающая неоднозначность компенсируется в полном выражении термодинамического потенциала за счет членов, содержаших прямые корреляции. Рассмотрим простейший способ введения параметра: $M_{s}(\cdot \mid t)=t M_{s}(\cdot)$. Поправка третьего порядка в таком представлении имеет вид $\left(\rho_{1}=\rho\right)$

$$
-\frac{\rho^{3}}{3 !} \int\left\{1+3 M_{2}+2 M_{2} M_{2}+\frac{1}{2} M_{2}(1,2) M_{2}(1,3) M_{2}(2,3)\right\} M_{3}(\{3\}) d\{3\} .
$$

Выражения $M_{2}$ и $M_{2} M_{2}$ означают симметризованные члены соответствующей структуры. Например, $3 M_{2} \Longleftrightarrow M_{2}(1,2)+M_{2}(1,3)+M_{2}(2,3)$.

Выражение для поправки четвертого порядка довольно громоздко, хотя и имеет достаточно простую структуру. Приведем алгоритм вычисления этой поправки. Воспользуемся тем, что в подынтегральном выражении координаты $(1),(2),(3)$ и функции $M_{2}(1,2), M_{2}(1,3), M_{2}(2,3)$ входят симметрично, и сопоставим этим функциям символ $\widetilde{M}$, произведению этих функций - соответствующую степень этого символа, функции $M_{3}(\{3\})$ - символ $\widetilde{M}_{3}$, функциям $M_{2}(i, 4)$ - символ $M$, функциям $M_{3}(i, k, 4)$ - символ $M_{3}$. Интеграл

$$
\begin{aligned}
& -\frac{\rho^{4}}{4 !} \int_{0}^{1} M_{4}(\{4\})(1+t M)^{3}\left(1+t M_{3}\right)^{3} \times \\
& \quad \times\left\{4(1+\widetilde{M})^{3}\left(1+\widetilde{M_{3}}\right)-3\left[(1+t \widetilde{M})^{3}+2(1-t) \widetilde{M}(1+t \widetilde{M})^{2}\right]\left(1+t \widetilde{M_{3}}\right)\right\} d t
\end{aligned}
$$

вычисляется элементарно. Заменяя использованные символы симметризованными произведениями соответствуюших функций и интегрируя по координатам $(1,2,3,4)$, получим поправку четвертого порядка. Эта поправка содержит более пятидесяти членов, приводить ее здесь нецелесообразно, но ее использование при численной реализации вариационной задачи трудностей не вызовет.

\section{Список литературы}

[1] Э. А. Аринштейн. ТМФ. 2000. Т. 124. № 1. С. 136-147.

[2] Э. А. Аринштейн, Р. М. Ганопольский. ТМФ. 2002. Т. 131. № 2. С. 278-287.

[3] Э. А. Аринштейн. ТМФ. 2004. Т. 141. № 1. С. 152-160.

Поступила в редакцию 21.VI.2004 г. 